\title{
Best Linear Unbiased Estimate using Buys-Ballot Procedure when Trend-Cycle Component is Linear
}

\author{
Iheanyi S. Iwueze \\ Department of Statistics \\ Federal University of Technology \\ Owerri, Imo State, Nigeria \\ isiwueze@yahoo.com \\ Eleazar C. Nwogu \\ Department of Statistics \\ Federal University of Technology \\ Owerri, Imo State, Nigeria \\ Jude C. Ajaraogu \\ Department of Mathematics and Statistics \\ Federal Polytechnic, Nekede, Owerri \\ Imo State, Nigeria
}

\begin{abstract}
The Best linear unbiased estimators of Buys-Ballot estimates when trend-cycle component is linear are discussed in this paper. The estimates are those proposed by Iwueze and Nwogu (2004). Discussed are the Chain Based Estimation method and the Fixed Based Estimation method. The variates for the Chain Based Estimation method were found to have constant mean and variance but are correlated with only one significant autocorrelation coefficient at lag one. The variates for the Fixed Based Estimation method were found to have constant mean, nonconstant variance but with constant autocorrelation coefficient at all lags. Best Linear unbiased estimators of the slope and intercept for the Chain Based Estimation variates only were derived since they exhibit stationarity. Numerical examples were used to illustrate the methods.
\end{abstract}

Keywords: Best linear unbiased Estimator, Buys-Ballot derived variables, stationarity, Minimum variance, Moving Average Process of order one.

\section{Introduction}

Iwueze and Nwogu (2004) developed two methods of estimating the parameters of a linear trend-cycle component from the periodic averages of the Buys-Ballot Table (Table 1). The procedure was initially developed for short period series in which the trend-cycle component $\left(\mathrm{M}_{\mathrm{t}}\right)$ is jointly estimated and can be represented by a linear equation:

$$
M_{t}=a+b t, t=1,2, \ldots, n
$$

The two alternative methods are: (i) the Chain Base Estimation (CBE) method which computes the slope from the relative periodic average changes and (ii) the Fixed Base Estimation (FBE) method which computes the slope using the first period as the base period for the periodic average changes. 
For short series in which the trend and cyclical components are jointly estimated, the two contending models for time series decomposition are the additive and multiplicative models (Chatfield (2004), Kendall and Ord (1990)).

Additive model:

$$
\begin{array}{ll}
\text { Additive model: } & \mathrm{X}_{\mathrm{t}}=\mathrm{M}_{\mathrm{t}}+\mathrm{S}_{\mathrm{t}}+\mathrm{e}_{\mathrm{t}} \\
\text { Multiplicative model: } & \mathrm{X}_{\mathrm{t}}=\mathrm{M}_{\mathrm{t}} \mathrm{S}_{\mathrm{t}} \mathrm{e}_{\mathrm{t}}
\end{array}
$$

Where for time $t, X_{t}$ is the observed series; $S_{t}$ is the seasonal component with the property that $\mathrm{S}_{(\mathrm{i}-1) \mathrm{s}+\mathrm{j}}=\mathrm{S}_{\mathrm{j}}, \mathrm{i}=1,2, \ldots, \mathrm{m}$, and $\mathrm{e}_{\mathrm{t}}$ is the irregular or random component. Results obtained by Iwueze and Nwogu (2004) for the additive and multiplicative models are summarized in Table 2.

It is clear from Table 2 that the trend-cycle estimates are the same for both the additive and multiplicative models. We can also note from Table 2 that estimates of the intercept (a) and the seasonal indices $\left(\mathrm{S}_{\mathrm{j}}, \mathrm{i}=1,2, \ldots, \mathrm{m}\right)$ depend on the estimate of the slope (b). This paper will therefore concentrate on the Best Linear Unbiased Estimator (BLUE) of the slope (b) parameter. For the additive model (1.2), it is assumed that the irregular/error component $\mathrm{e}_{\mathrm{t}}$ is the Gaussian $\mathrm{N}\left(0, \sigma_{1}^{2}\right)$ white noise, while for the multiplicative model (1.3), $\mathrm{e}_{\mathrm{t}}$ is the Gaussian $\mathrm{N}\left(1, \sigma_{2}^{2}\right)$ white noise. For the additive model (1.2), the assumption is that the sum of the seasonal component over a complete period is zero $\left(\sum_{j=0}^{s} S_{j}=0\right)$, while for the multiplicative model (1.3), the sum of the seasonal component over a complete period is $\mathrm{s}\left(\sum_{\mathrm{j}=0}^{\mathrm{s}} \mathrm{S}_{\mathrm{j}}=\mathrm{s}\right)$.

The multiplicative model (1.3) can be linearized to become the additive model (1.4).

$$
X_{t}^{*}=M_{t}^{*}+S_{t}^{*}+e_{t}^{*}, t=1,2, \ldots, n
$$

where $X_{t}^{*}=\log _{e} X_{t}, M_{t}^{*}=\log _{e} M_{t}, S_{t}^{*}=\log _{e} S_{t}, e_{t}^{*}=\log _{e} e_{t}$. The behaviour of $M_{t}^{*}=\log _{e} M_{t}$ when $M_{t}$ is represented by a linear equation (1.1) have been studied by Iwueze and Akpanta (2007) and it was shown that for $-0.01 \leq \mathrm{b} / \mathrm{a} \leq 0.06, \quad \mathrm{M}_{\mathrm{t}}^{*}$ could still be represented by a straight line $\mathrm{M}_{\mathrm{t}}^{*}=\alpha+\beta \mathrm{t}$, with $\alpha=\log _{\mathrm{e}}$ a and $\beta=\mathrm{b} / \mathrm{a}$. The behaviour of $\mathrm{S}_{\mathrm{t}}^{*}=\log _{\mathrm{e}} \mathrm{S}_{\mathrm{t}}$ to achieve $\sum_{\mathrm{j}=0}^{\mathrm{s}} \mathrm{S}_{\mathrm{j}}=\mathrm{s}$ have been studied by Iwueze et al (2008). The behaviour of $\mathrm{e}_{\mathrm{t}}^{*}=\log _{\mathrm{e}} \mathrm{e}_{\mathrm{t}}$ for $\mathrm{e}_{\mathrm{t}}^{*} \sim \mathrm{N}\left(0, \sigma^{2}\right)$ when $\mathrm{e}_{\mathrm{t}} \sim \mathrm{N}\left(1, \sigma^{2}\right)$ have been studied by Iwueze (2007) and it was shown that the logarithmic transform of the lefttruncated $\mathrm{N}\left(1, \sigma^{2}\right)$ distribution is approximately normal when $\sigma<0.1$. It follows 
that we can study the additive model (1.1) and apply the results obtain to the multiplicative model after linearization.

The main objective of this paper is to obtain the BLUE of the slope parameter for the additive model. Section 2 presents the covariance structure of CBE derived variables, while Section 3 presents the covariance structure of the FBE derived variables. Section 4 contains the determination of the BLUE for the CBE estimate of the slope parameter. Section 5 presents the simple average of the CBE derived variables, Section 6 contains the numerical examples while Section 7 contains the concluding remarks.

Table 1: Buys-Ballot Table

\begin{tabular}{|c|c|c|c|c|c|c|c|c|c|}
\hline \multirow{2}{*}{$\mathrm{p}$} & \multicolumn{9}{|c|}{ Season } \\
\cline { 2 - 10 } & 1 & 2 & $\cdots$ & $\mathrm{j}$ & $\cdots$ & $\mathrm{s}$ & $\mathrm{T}_{\mathrm{i} .}$ & $\overline{\mathrm{X}}_{\mathrm{i} .}$ & $\hat{\sigma}_{\mathrm{i} .}$ \\
\hline 1 & $\mathrm{X}_{1}$ & $\mathrm{X}_{2}$ & $\cdots$ & $\mathrm{X}_{\mathrm{j}}$ & $\cdots$ & $\mathrm{X}_{\mathrm{s}}$ & $\mathrm{T}_{1 .}$ & $\overline{\mathrm{X}}_{1 .}$ & $\hat{\sigma}_{1 .}$ \\
\hline 2 & $\mathrm{X}_{\mathrm{s}+1}$ & $\mathrm{X}_{\mathrm{s}+2}$ & $\cdots$ & $\mathrm{X}_{\mathrm{s}+\mathrm{j}}$ & $\cdots$ & $\mathrm{X}_{2 \mathrm{~s}}$ & $\mathrm{~T}_{2 .}$ & $\overline{\mathrm{X}}_{2 .}$ & $\hat{\sigma}_{2 .}$ \\
\hline 3 & $\mathrm{X}_{2 \mathrm{~s}+1}$ & $\mathrm{X}_{2 \mathrm{~s}+2}$ & $\cdots$ & $\mathrm{X}_{2 \mathrm{~s}+\mathrm{j}}$ & $\cdots$ & $\mathrm{X}_{3 \mathrm{~s}}$ & $\mathrm{~T}_{3 .}$ & $\overline{\mathrm{X}}_{3 .}$ & $\hat{\sigma}_{3 .}$ \\
\hline$\ldots$ & $\ldots$ & $\ldots$ & $\cdots$ & $\ldots$ & $\cdots$ & $\ldots$ & $\ldots$ & $\ldots$ & $\ldots$ \\
\hline $\mathrm{i}$ & $\mathrm{X}_{(\mathrm{i}-1) \mathrm{s}+1}$ & $\mathrm{X}_{(\mathrm{i}-1) \mathrm{s}+2}$ & $\cdots$ & $\mathrm{X}_{(\mathrm{i}-1) \mathrm{s}+\mathrm{j}}$ & $\cdots$ & $\mathrm{X}_{(\mathrm{i}-1) \mathrm{s}+\mathrm{s}}$ & $\mathrm{T}_{\mathrm{i} .}$ & $\overline{\mathrm{X}}_{\mathrm{i} .}$ & $\hat{\sigma}_{\mathrm{i} .}$ \\
\hline$\ldots$ & $\ldots$ & $\ldots$ & $\cdots$ & $\ldots$ & $\cdots$ & $\ldots$ & $\ldots$ & $\ldots$ & $\ldots$ \\
\hline $\mathrm{m}$ & $\mathrm{X}_{(\mathrm{m}-1) \mathrm{s}+1}$ & $\mathrm{X}_{(\mathrm{m}-1) \mathrm{s}+2}$ & $\cdots$ & $\mathrm{X}_{(\mathrm{m}-1) \mathrm{s}+\mathrm{j}}$ & $\cdots$ & $\mathrm{X}_{\mathrm{ms}}$ & $\mathrm{T}_{\mathrm{m} .}$ & $\overline{\mathrm{X}}_{\mathrm{m} .}$ & $\hat{\sigma}_{\mathrm{m} .}$ \\
\hline $\mathrm{T}_{. \mathrm{j}}$ & $\mathrm{T}_{.1}$ & $\mathrm{~T}_{.2}$ & $\cdots$ & $\mathrm{T}_{. \mathrm{j}}$ & $\cdots$ & $\mathrm{T}_{. \mathrm{s}}$ & $\mathrm{T}_{. .}$ & & \\
\hline$\overline{\mathrm{X}}_{. \mathrm{j}}$ & $\overline{\mathrm{X}}_{.1}$ & $\overline{\mathrm{X}}_{.2}$ & $\cdots$ & $\overline{\mathrm{X}}_{. \mathrm{j}}$ & $\cdots$ & $\mathrm{X}_{\mathrm{s}}$ & & $\overline{\mathrm{X}}_{. .}$ & \\
\hline$\hat{\sigma}_{. \mathrm{j}}$ & $\hat{\sigma}_{.1}$ & $\hat{\sigma}_{.2}$ & $\cdots$ & $\hat{\sigma}_{. \mathrm{j}}$ & $\cdots$ & $\hat{\sigma}_{. \mathrm{s}}$ & & & $\hat{\sigma}_{. .}$ \\
\hline
\end{tabular}

$\mathrm{T}_{\mathrm{i} .}=\sum_{\mathrm{j}=1}^{\mathrm{s}} \mathrm{X}_{(\mathrm{i}-1) \mathrm{s}+\mathrm{j}}, \mathrm{i}=1,2, \ldots, \mathrm{m} ; \overline{\mathrm{X}}_{\mathrm{i} .}=\frac{\mathrm{T}_{\mathrm{i} .}}{\mathrm{s}}=\frac{1}{\mathrm{~s}} \sum_{\mathrm{j}=1}^{\mathrm{s}} \mathrm{X}_{(\mathrm{i}-1) \mathrm{s}+\mathrm{j}}, \mathrm{i}=1,2, \ldots, \mathrm{m}$;

$T_{. j}=\sum_{i=1}^{m} X_{(i-1) s+j}, j=1,2, \ldots, s$

$\bar{X}_{. j}=\frac{T_{. j}}{m}=\frac{1}{m} \sum_{i=1}^{m} X_{(i-1) s+j}, j=1,2, \ldots, s ;$

$\mathrm{T}_{. .}=\sum_{\mathrm{i}=1}^{\mathrm{m}} \mathrm{T}_{\mathrm{i} .}=\sum_{\mathrm{j}=1}^{\mathrm{s}} \mathrm{T}_{. \mathrm{j}}=\sum_{\mathrm{i}=1}^{\mathrm{m}} \sum_{\mathrm{j}=1}^{\mathrm{s}} \mathrm{X}_{(\mathrm{i}-1) \mathrm{s}+\mathrm{j}} ; \overline{\mathrm{X}}_{. .}=\frac{\mathrm{T}_{. .}}{\mathrm{ms}}=\frac{\mathrm{T}_{. .}}{\mathrm{ms}}, \mathrm{n}=\mathrm{ms}$

$\hat{\sigma}_{i .}=\sqrt{\frac{1}{s-1} \sum_{j=1}^{s}\left(X_{(i-1) s+j}-\bar{X}_{i .}\right)^{2}} \quad, i=1,2, \ldots, m$;

$\hat{\sigma}_{. j}=\sqrt{\frac{1}{m-1} \sum_{i=1}^{m}\left(X_{(i-1) s+j}-\bar{X}_{. j}\right)^{2}}, j=1,2, \ldots, s$ 


$$
\hat{\sigma}=\sqrt{\frac{1}{n-1} \sum_{i=1}^{m} \sum_{j=1}^{s}\left(X_{(i-1) s+j}-\bar{X}_{. .}\right)^{2}}
$$

Table 2: Buys-Ballot estimates for linear trend.

\begin{tabular}{|c|c|c|}
\hline & Additive model (1.2) & Multiplicative model (1.3) \\
\hline $\mathrm{T}_{\mathrm{i}}$ & $\mathrm{as}+\frac{\mathrm{bs}}{2}[(2 \mathrm{i}-1) \mathrm{s}+1]$ & $\mathrm{as}+\frac{\mathrm{bs}}{2}[(2 \mathrm{i}-1) \mathrm{s}+1]$ \\
\hline$\overline{\mathrm{X}}_{\mathrm{i}}$ & $a+\frac{b}{2}[(2 i-1) s+1]$ & $a+\frac{b}{2}[(2 i-1) s+1]$ \\
\hline $\mathrm{T}_{. \mathrm{j}}$ & $\mathrm{ma}+\frac{\mathrm{mb}}{2}[2 \mathrm{j}+\mathrm{n}-\mathrm{s}]+\mathrm{mS}_{\mathrm{j}}$ & {$\left[\mathrm{ma}+\frac{\mathrm{mb}}{2}(2 \mathrm{j}+\mathrm{n}-\mathrm{s})\right] \mathrm{S}_{\mathrm{j}}$} \\
\hline$\overline{\mathrm{X}}_{. j}$ & $a+\frac{b}{2}[2 j+n-s]+S_{j}$ & {$\left[a+\frac{b}{2}(2 j+n-s)\right] S_{j}$} \\
\hline $\mathrm{T}_{\text {. }}$ & $\mathrm{na}+\frac{\mathrm{nb}}{2}[\mathrm{n}+1]$ & $\mathrm{na}+\frac{\mathrm{nb}}{2}[\mathrm{n}+1]$ \\
\hline$\overline{\mathrm{X}}$. & $\mathrm{a}+\frac{\mathrm{b}}{2}[\mathrm{n}+1]$ & $\mathrm{a}+\frac{\mathrm{b}}{2}[\mathrm{n}+1]$ \\
\hline$\hat{\mathrm{b}}(\mathrm{CBE})$ & $\frac{\overline{\mathrm{X}}_{\mathrm{m} .}-\overline{\mathrm{X}}_{1}}{\mathrm{n}-\mathrm{s}}$ & $\frac{\bar{X}_{m .}-\bar{X}_{1}}{n-s}$ \\
\hline$\hat{\mathrm{b}}(\mathrm{FBE})$ & $\frac{1}{n-s} \sum_{i=2}^{m}\left(\frac{\bar{X}_{i .}-\bar{X}_{1 .}}{i-1}\right)$ & $\frac{1}{n-s} \sum_{i=2}^{m}\left(\frac{\bar{X}_{i .}-\bar{X}_{1 .}}{i-1}\right)$ \\
\hline$\hat{\mathrm{a}}$ & $\bar{X}_{. .}-\frac{\hat{b}}{2}[\mathrm{n}+1]$ & $\bar{X}_{. .}-\frac{\hat{b}}{2}[n+1]$ \\
\hline $\mathrm{S}_{\mathrm{j}}$ & $\bar{X}_{. j}-\left[\bar{X}_{. .}+\frac{\hat{b}}{2}(2 j-s-1)\right.$ & $\bar{X}_{. j} /\left[\bar{X}_{. .}+\frac{\hat{b}}{2}(2 j-s-1)\right.$ \\
\hline
\end{tabular}

\section{Covariance Analysis of the CBE Derived Variables: Additive Model}

Under the CBE method, the estimate of the slope $(\hat{b})$ was calculated as the average of $\hat{b}_{i}^{(c)}, i=1,2, \ldots, m-1$ given by Iwueze and Nwogu (2004) as:

$$
\hat{b}_{i}^{(c)}=\frac{\bar{X}_{(i+1) .}-\bar{X}_{i .}}{s}, i=1,2, \ldots, m-1
$$

As noted earlier for the additive model, the irregular components are independently, identically and normally distributed with mean zero and common 
variance $\sigma_{1}^{2}=\sigma^{2}$. Under this assumption, $\overline{\mathrm{e}}_{\mathrm{i} .} \sim \mathrm{N}\left(0, \frac{\sigma^{2}}{\mathrm{~s}}\right), \overline{\mathrm{e}}_{. \mathrm{j}} \sim \mathrm{N}\left(0, \frac{\sigma^{2}}{\mathrm{~m}}\right)$, $\overline{\mathrm{e}}_{. .} \sim \mathrm{N}\left(0, \frac{\sigma^{2}}{\mathrm{n}}\right)$

Using (1.2), the periodic averages are given by

$$
\bar{X}_{i .}=a+\frac{b}{2}[(2 i-1) s+1]+\bar{e}_{i,}, i=1,2, \ldots, m
$$

Hence, our variable of interest is now given by

$$
\hat{\mathrm{b}}_{\mathrm{i}}^{(\mathrm{c})}=\frac{1}{\mathrm{~s}}\left(\overline{\mathrm{X}}_{(\mathrm{i}+1) .}-\overline{\mathrm{X}}_{\mathrm{i} .}\right)=\mathrm{b}+\frac{1}{\mathrm{~s}}\left(\overline{\mathrm{e}}_{(\mathrm{i}+1) .}-\overline{\mathrm{e}}_{\mathrm{i} .}\right), \mathrm{i}=1,2, \ldots, \mathrm{m}-1
$$

Therefore, the expected value and variance of $b_{i}^{(c)}$ are

$$
\begin{aligned}
E\left(\hat{b}_{i}^{(c)}\right) & =E(b)+\frac{1}{s} E\left(\bar{e}_{(i+1)}-\bar{e}_{i .}\right)=b \\
\operatorname{var}\left(\hat{b}_{i}^{(c)}\right) & =\sigma_{\hat{b}_{i}^{(c)}}^{2}=E\left[\left(\hat{b}_{i}^{(c)}-b\right)^{2}\right]=\frac{1}{s^{2}} E\left[\left(\bar{e}_{(i+1) .}-\bar{e}_{i .}\right)^{2}\right] \\
& =\frac{2 \sigma^{2}}{s^{3}}
\end{aligned}
$$

The covariance between $b_{i}^{(c)}$ and $b_{j}^{(c)}$ is

$$
\begin{aligned}
& \operatorname{cov}\left(\hat{b}_{i}^{(c)}, \hat{b}_{j}^{(c)}\right)=\sigma_{i j}=E\left[\left(\hat{b}_{i}^{(c)}-E\left(\hat{b}_{i}^{(c)}\right)\right)\left(\hat{b}_{j}^{(c)}-E\left(\hat{b}_{j}^{(c)}\right)\right)\right] \\
& =\frac{1}{\mathrm{~s}^{2}} \mathrm{E}\left[\left(\overline{\mathrm{e}}_{(\mathrm{i}+1) .}-\overline{\mathrm{e}}_{\mathrm{i} .}\right)\left(\overline{\mathrm{e}}_{(\mathrm{j}+1) .}-\overline{\mathrm{e}}_{\mathrm{j} .}\right)\right] \\
& =\frac{1}{\mathrm{~s}^{2}} \mathrm{E}\left[\overline{\mathrm{e}}_{(\mathrm{i}+1) \cdot} \overline{\mathrm{e}}_{(\mathrm{j}+1) \cdot}-\overline{\mathrm{e}}_{(\mathrm{i}+1) \cdot} \overline{\mathrm{e}}_{\mathrm{j} .}-\overline{\mathrm{e}}_{\mathrm{i} .} \overline{\mathrm{e}}_{(\mathrm{j}+1) .}+\overline{\mathrm{e}}_{\mathrm{i} .} \overline{\mathrm{e}}_{\mathrm{j} .}\right]
\end{aligned}
$$

For $\mathrm{j}=\mathrm{i}+1$,

$$
\sigma_{i j}=\frac{-1}{s^{2}} E\left(\bar{e}_{(I+1) .}^{2}\right)=\frac{-1}{s^{2}}\left(\frac{\sigma^{2}}{s}\right)=\frac{-\sigma^{2}}{s^{3}}
$$

For $\mathrm{j}=\mathrm{i}-1$,

$$
\sigma_{i j}=\frac{-1}{s^{2}} E\left(\bar{e}_{i .}^{2}\right)=\frac{-1}{s^{2}}\left(\frac{\sigma^{2}}{s}\right)=\frac{-\sigma^{2}}{s^{3}}
$$

For $\mathrm{j}=\mathrm{i} \pm \mathrm{k}, \mathrm{k}>1$,

$$
\sigma_{i j}=0
$$


In summary, let $\mathrm{R}(\mathrm{k})=\operatorname{cov}\left(\hat{\mathrm{b}}_{\mathrm{i} .}^{(\mathrm{c})}, \hat{\mathrm{b}}_{(\mathrm{i}-\mathrm{k})}^{(\mathrm{c})}\right)$ and $\rho_{\mathrm{k}}=\mathrm{R}(\mathrm{k}) / \mathrm{R}(0)$. The results (2.5) through (2.9) can be summarized as follows:

$$
\begin{gathered}
\mathrm{R}(\mathrm{k})=\left\{\begin{array}{cc}
2 \sigma^{2} / \mathrm{s}^{3}, \mathrm{k}=0 \\
-\sigma^{2} / \mathrm{s}^{3}, \mathrm{k}= \pm 1 \\
0, & \mathrm{k}>1
\end{array}\right. \\
\rho_{\mathrm{k}}=\left\{\begin{array}{cc}
1, & \mathrm{k}=0 \\
-1 / 2, \mathrm{k}= \pm 1 \\
0, & \mathrm{k}>1
\end{array}\right.
\end{gathered}
$$

We have shown that the sequence, $\hat{b}_{i}^{(c)}, i=1,2, \ldots, m-1$, of CBE derived variables have the covariance structure of a moving average process of order one $(\mathrm{MA}(1))$. For more details on MA(1) processes, see Box et al, (1994), Chatfield (2004).

\section{Covariance Analysis of the FBE Derived Variables: Additive Model}

Under the FBE method, the estimate of the slope $(\hat{b})$ was calculated as the average of $\hat{b}_{i}^{(f)}, i=1,2, \ldots, m-1$ given by Iwueze and Nwogu (2004) as:

$$
\hat{b}_{i}^{(f)}=\frac{\bar{X}_{(i+1) .}-\bar{X}_{1 .}}{(i-1) s}, i=1,2, \ldots, m-1
$$

Using (1.2),

$$
\hat{b}_{i}^{(f)}=\frac{\bar{X}_{(i+1) .}-\bar{X}_{1 .}}{(i-1) s}=b+\frac{\bar{e}_{(i+1) .}-\bar{e}_{1 .}}{(i-1) s}, i=1,2, \ldots, m-1
$$

Hence, the expected value and variance of $b_{i}^{(f)}$ are

$$
\begin{aligned}
& E\left(\hat{b}_{i}^{(f)}\right)=E(b)+\frac{1}{(i-1) s} E\left(\bar{e}_{(i+1) .}-\bar{e}_{1 .}\right)=b \\
& \operatorname{var}\left(\hat{b}_{i}^{(f)}\right)=\sigma_{\hat{b}_{i}^{(f)}}^{2}=E\left[\left(\hat{b}_{i}^{(f)}-b\right)^{2}\right]=\frac{1}{(i-1)^{2} s^{2}} E\left[\left(\bar{e}_{(i+1) .}-\bar{e}_{1 .}\right)^{2}\right] \\
&=\frac{2 \sigma^{2}}{(i-1)^{2} s^{3}} \\
& \operatorname{cov}\left(\hat{b}_{i}^{(f)}, \hat{b}_{j}^{(f)}\right)=\sigma_{i j}=E\left[\left(\hat{b}_{i}^{(f)}-E\left(\hat{b}_{i}^{(f)}\right)\right)\left(\hat{b}_{j}^{(f)}-E\left(\hat{b}_{j}^{(f)}\right)\right)\right] \\
&=E\left[\left(\hat{b}_{i}^{(f)}-b\right)\left(\hat{b}_{j}^{(f)}-b\right)\right] \\
&= \frac{1}{(i-1)(j-1) s^{2}} E\left[\left(\bar{e}_{(i+1) \cdot}-\bar{e}_{1 .}\right)\left(\bar{e}_{(j+1) \cdot}-\bar{e}_{1 .}\right)\right] \\
&=\frac{1}{(i-1)(j-1) s^{2}} E\left[\bar{e}_{(i+1) \cdot} \bar{e}_{(j+1) .}-\bar{e}_{(i+1) \cdot} \bar{e}_{1 .}-\bar{e}_{i .} \bar{e}_{(j+1) .}+\bar{e}_{1 .}^{2}\right]
\end{aligned}
$$


For $\mathrm{j}=\mathrm{i}+\mathrm{k}$,

$$
\sigma_{i j}=\frac{1}{(i-1)(j-1) s^{2}} E\left(\bar{e}_{1 .}^{2}\right)=\frac{\sigma^{2}}{(i-1)(j-1) s^{2}}
$$

Hence, the autocovariance and autocorrelation structures are:

$$
\begin{aligned}
R(k) & =\left\{\begin{array}{l}
\frac{2 \sigma^{2}}{(i-1)^{2} s^{3}}, k=0 \\
\frac{\sigma^{2}}{(i-1)(j-1) s^{3}}, k \neq 0
\end{array}\right. \\
\rho_{k} & = \begin{cases}1, & k=0 \\
1 / 2, & k= \pm 1, \pm 2, \ldots\end{cases}
\end{aligned}
$$

We have shown that the sequence, $\hat{b}_{i}^{(f)}, i=1,2, \ldots, m-1$, of FBE derived variables are not stationary and their average as an estimate of slope (b), will not give a reliable estimate in its present state.

\section{Best linear unbiased estimate of slope (b) using the CBE derived variables}

The CBE derived variables $\left(\hat{b}_{i}^{(c)}, i=1,2, \ldots, m-1\right)$ have been shown to be stationary and can be used for estimation, while the FBE derived variables $\left(\hat{b}_{i}^{(f)}, i=1,2, \ldots, m-1\right)$ are not stationary and estimates based on them will not be reliable. The sequence of $\mathrm{CBE}$ derived random variables $\hat{b}_{i}^{(c)}, i=1,2, \ldots, m-1$, are found to have the covariance structure of a first-order moving average process (MA(1) process) with the autocorrelation function given by (2.11).

Let $\mathrm{a}_{1}, \mathrm{a}_{2}, \mathrm{a}_{\mathrm{m}-1}$ be any set of real numbers. A linear estimate of the mean $\mathrm{b}=\mathrm{E}\left(\hat{\mathrm{b}}_{\mathrm{i}}^{(\mathrm{c})}\right)$ is given by

$$
\mathrm{T}=\sum_{\mathrm{t}=1}^{\mathrm{m}-1} \mathrm{a}_{\mathrm{i}} \hat{\mathrm{b}}_{\mathrm{i}}^{(\mathrm{c})}
$$

If $\mathrm{T}$ is unbiased, we obtain that

$$
E(T)=\sum_{t=1}^{m-1} a_{i} E\left(\hat{b}_{i}^{(c)}\right)=\sum_{t=1}^{m-1} a_{i} b=b \sum_{t=1}^{m-1} a_{i}=b
$$

$\mathrm{T}$ is unbiased if and only if

$$
\sum_{\mathrm{t}=1}^{\mathrm{m}-1} \mathrm{a}_{\mathrm{i}}=1
$$


The variance of $\mathrm{T}$ is given by

$$
\operatorname{var}(\mathrm{T})=\sum_{\mathrm{i}=1}^{\mathrm{m}-1} \mathrm{a}_{\mathrm{i}}^{2} \operatorname{var}\left(\mathrm{Y}_{\mathrm{i}}\right)+2 \sum_{\mathrm{i}} \sum_{\mathrm{J}} \mathrm{a}_{\mathrm{i}} \mathrm{a}_{\mathrm{j}} \operatorname{cov}\left(\mathrm{Y}_{\mathrm{i}}, \mathrm{Y}_{\mathrm{j}}\right)
$$

For the second order stationary sequence of random variables $\hat{b}_{i}^{(c)}, i=1,2, \ldots, m-1$, with autocorrelation structure $(2.11)$, $\operatorname{var}(T)$ can be written as

$$
\begin{aligned}
\operatorname{var}(\mathrm{T}) & =\mathrm{R}(0) \sum_{\mathrm{i}=1}^{\mathrm{m}-1} \mathrm{a}_{\mathrm{i}}^{2}+2 \mathrm{R}(1) \sum_{\mathrm{i}=1}^{\mathrm{m}-2} \mathrm{a}_{\mathrm{i}} \mathrm{a}_{\mathrm{i}+1} \\
& =\mathrm{R}(0)\left\{\sum_{\mathrm{i}=1}^{\mathrm{m}-1} \mathrm{a}_{\mathrm{i}}^{2}+2 \rho_{1} \sum_{\mathrm{i}=1}^{\mathrm{m}-2} \mathrm{a}_{\mathrm{i}} \mathrm{a}_{\mathrm{i}+1}\right\} \\
= & \mathrm{R}(0)\left\{\sum_{\mathrm{i}=1}^{\mathrm{m}-1} \mathrm{a}_{\mathrm{i}}^{2}-\sum_{\mathrm{i}=1}^{\mathrm{m}-2} \mathrm{a}_{\mathrm{i}} \mathrm{a}_{\mathrm{i}+1}\right\} \\
= & \left(\frac{2 \sigma^{2}}{\mathrm{~s}^{3}}\right)\left\{\sum_{\mathrm{i}=1}^{\mathrm{m}-1} \mathrm{a}_{\mathrm{i}}^{2}-\sum_{i=1}^{\mathrm{m}-2} \mathrm{a}_{\mathrm{i}} \mathrm{a}_{\mathrm{i}+1}\right\}
\end{aligned}
$$

Linear unbiased estimates of $b$ that have minimum variance (among all linear unbiased estimates) are called best linear unbiased estimates (BLUE.s). Let

$$
S(\mathbf{a})=\sum_{i=1}^{m-1} a_{i}^{2}-\sum_{i=1}^{m-2} a_{i} a_{i+1}
$$

From (4.6), $\min (\operatorname{var}(\mathrm{T}))=\mathrm{R}(0) \min (\mathrm{S}(\mathbf{a}))$. Hence, the BLUE of $\mathrm{b}$ is obtained if we choose $\mathrm{a}_{1}, \mathrm{a}_{2}, \mathrm{a}_{\mathrm{m}-1}$ that minimize $\mathrm{S}(\mathbf{a})$ with respect to the constraint $\sum_{t=1}^{m-1} a_{t}=1$. However, when $\rho_{k}=0$, for all $k, a_{i}=\frac{1}{m-1}$ (see Rohatgi (1976)).

As an example of the minimization of (4.7) subject to the constraint $\sum_{\mathrm{i}=1}^{\mathrm{m}-1} \mathrm{a}_{\mathrm{i}}=1$, we let $\mathrm{m}-1=10 \Rightarrow \mathrm{m}=11$. Equation (4.7) reduces to

$$
\begin{aligned}
S(a)= & a_{1}^{2}+a_{2}^{2}+a_{3}^{2}+a_{4}^{2}+a_{5}^{2}+a_{6}^{2}+a_{7}^{2}+a_{8}^{2}+a_{9}^{2} \\
& +\left(1-a_{1}-a_{2}-a_{3}-a_{4}-a_{5}-a_{6}-a_{7}-a_{8}-a_{9}\right)^{2} \\
& -a_{1} a_{2}-a_{2} a_{3}-a_{3} a_{4}-a_{4} a_{5}-a_{5} a_{6}-a_{6} a_{7}-a_{8} a_{9} \\
& -a_{9}\left(1-a_{1}-a_{2}-a_{3}-a_{4}-a_{5}-a_{6}-a_{7}-a_{8}-a_{9}\right)
\end{aligned}
$$


By equating $\partial \mathrm{S}(\mathbf{a}) / \partial \mathrm{a}_{\mathrm{j}}=0$, we obtain the system of linear equations given in (4.9).

$$
\begin{array}{r}
4 a_{1}+a_{2}+2 a_{3}+2 a_{4}+2 a_{5}+2 a_{6}+2 a_{7}+2 a_{8}+3 a_{9}=2 \\
a_{1}+4 a_{2}+a_{3}+2 a_{4}+2 a_{5}+2 a_{6}+2 a_{7}+2 a_{8}+3 a_{9}=2 \\
2 a_{1}+a_{2}+4 a_{3}+a_{4}+2 a_{5}+2 a_{6}+2 a_{7}+2 a_{8}+3 a_{9}=2 \\
2 a_{1}+2 a_{2}+a_{3}+4 a_{4}+a_{5}+2 a_{6}+2 a_{7}+2 a_{8}+3 a_{9}=2 \\
2 a_{1}+2 a_{2}+2 a_{3}+a_{4}+4 a_{5}+a_{6}+2 a_{7}+2 a_{8}+3 a_{9}=2 \\
2 a_{1}+2 a_{2}+2 a_{3}+2 a_{4}+a_{5}+4 a_{6}+a_{7}+2 a_{8}+3 a_{9}=2 \\
2 a_{1}+2 a_{2}+2 a_{3}+2 a_{4}+2 a_{5}+a_{6}+4 a_{7}+a_{8}+3 a_{9}=2 \\
2 a_{1}+2 a_{2}+2 a_{3}+2 a_{4}+2 a_{5}+2 a_{6}+a_{7}+4 a_{8}+2 a_{9}=2 \\
3 a_{1}+3 a_{2}+3 a_{3}+3 a_{4}+3 a_{5}+3 a_{6}+3 a_{7}+2 a_{8}+6 a_{9}=3
\end{array}
$$

We put the system of linear equations (4.9) in matrix form, to obtain

$$
\left(\begin{array}{l}
\mathrm{a}_{1} \\
\mathrm{a}_{2} \\
\mathrm{a}_{3} \\
\mathrm{a}_{4} \\
\mathrm{a}_{5} \\
\mathrm{a}_{6} \\
\mathrm{a}_{7} \\
\mathrm{a}_{8} \\
\mathrm{a}_{9}
\end{array}\right)=\left(\begin{array}{lllllllll}
4 & 1 & 2 & 2 & 2 & 2 & 2 & 2 & 3 \\
1 & 4 & 1 & 2 & 2 & 2 & 2 & 2 & 3 \\
2 & 1 & 4 & 1 & 2 & 2 & 2 & 2 & 3 \\
2 & 2 & 1 & 4 & 1 & 2 & 2 & 2 & 3 \\
2 & 2 & 2 & 1 & 4 & 1 & 2 & 2 & 3 \\
2 & 2 & 2 & 2 & 1 & 4 & 1 & 2 & 3 \\
2 & 2 & 2 & 2 & 2 & 1 & 4 & 1 & 3 \\
2 & 2 & 2 & 2 & 2 & 2 & 1 & 4 & 2 \\
3 & 3 & 3 & 3 & 3 & 3 & 3 & 2 & 6
\end{array}\right)^{-1}\left(\begin{array}{l}
2 \\
2 \\
2 \\
2 \\
2 \\
2 \\
2 \\
2 \\
3
\end{array}\right)
$$

Evaluating (4.10) with $\mathrm{a}_{10}=1-\mathrm{a}_{1}-\mathrm{a}_{2}-\mathrm{a}_{3}-\mathrm{a}_{4}-\mathrm{a}_{5}-\mathrm{a}_{6}-\mathrm{a}_{7}-\mathrm{a}_{8}-\mathrm{a}_{9}$, we obtained the following weights:

$$
\begin{aligned}
& \mathrm{a}_{1}=0.046 ; \mathrm{a}_{2}=0.082 \mathrm{a}_{3}=0.109 ; \mathrm{a}_{4}=0.127 ; \mathrm{a}_{5}=0.136 ; \mathrm{a}_{7}=0.127 ; \\
& \mathrm{a}_{8}=0.109 ; \mathrm{a}_{9}=0.082 ; \mathrm{a}_{10}=0.046 ; \mathrm{S}(\mathbf{a})=0.005 .
\end{aligned}
$$

Given in Table 3 are the weights for $\mathrm{m}=3,4, \ldots, 21(\mathrm{~m}-1=2,3, \ldots, 20)$. The plot of $\mathrm{S}(\mathbf{a})$ against $\mathrm{m}$ is given in Figure 1. Also illustrated in Figure 1 is the fact that $\mathrm{S}($ a $)$ follows an exponential distribution (Draper and Smith, 1999) given by

$$
\mathrm{S}(\mathbf{a})=\mathrm{e}^{0.2862-0.7156 \mathrm{~m}+0.0177 \mathrm{~m}^{2}} ; \mathrm{R}^{2}=0.99
$$




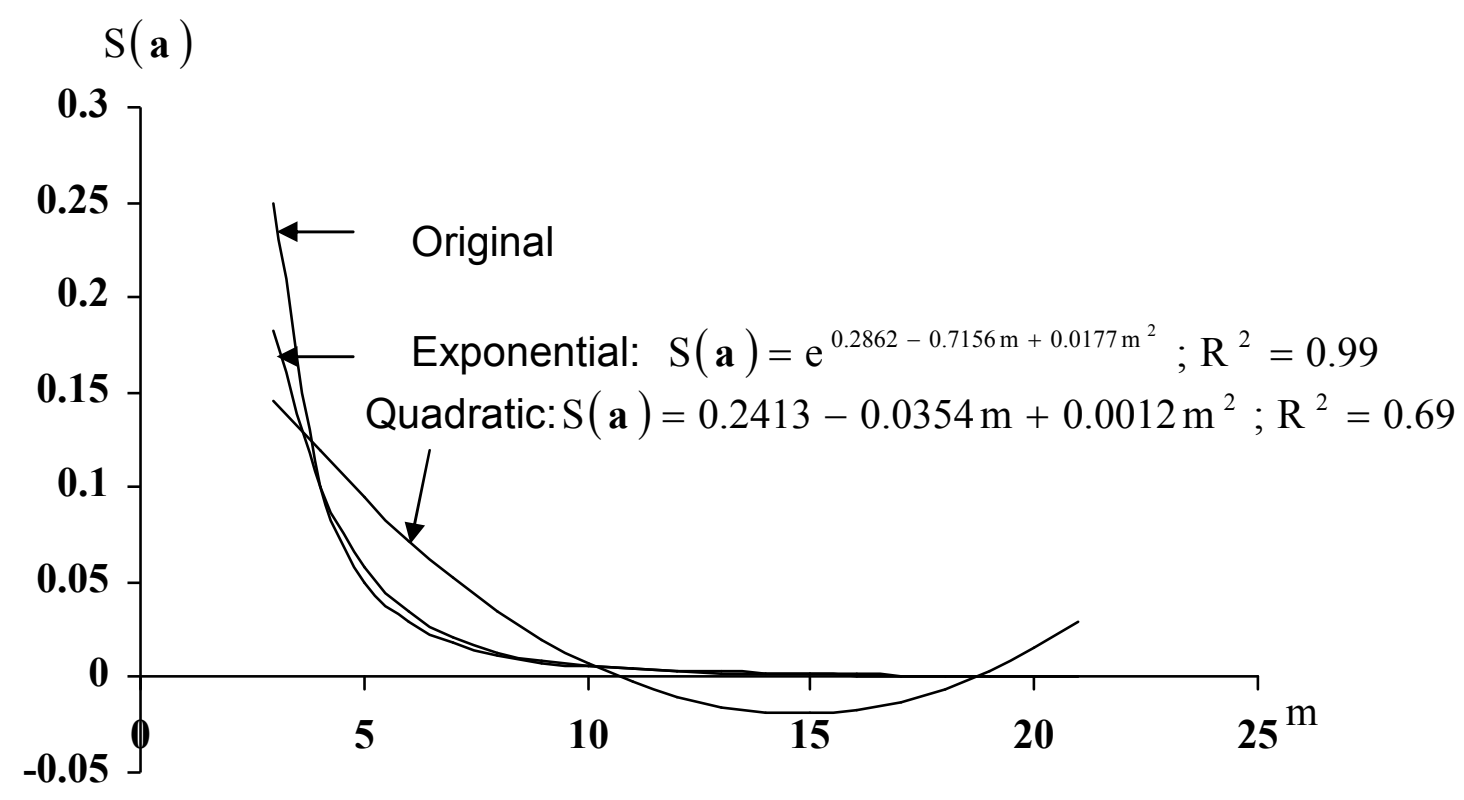

Figure 1: Plot of $\mathrm{S}(\mathbf{a})$ against $\mathrm{m}$

Table 3: Sample sizes $(m)$ and their corresponding weights $\left(a_{i}, i=1,2, \ldots, m-1\right)$

\begin{tabular}{|c|c|c|c|c|c|c|c|c|c|c|c|c|c|c|c|c|c|c|c|}
\hline \multirow{2}{*}{$a_{i}$} & \multicolumn{19}{|c|}{ Sample size $=m$} \\
\hline & 3 & 4 & 5 & 6 & 7 & 8 & 9 & 10 & 11 & 12 & 13 & 14 & 15 & 16 & 17 & 18 & 19 & 20 & 21 \\
\hline$a_{1}$ & 0.500 & 0.300 & 0.200 & 0.143 & 0.107 & 0.083 & 0.067 & 0.055 & 0.046 & 0.039 & 0.034 & 0.029 & 0.025 & 0.022 & 0.019 & 0.017 & 0.016 & 0.014 & 0.013 \\
\hline$a_{2}$ & 0.500 & 0.400 & 0.300 & 0.229 & 0.179 & 0.143 & 0.117 & 0.097 & 0.082 & 0.070 & 0.060 & 0.052 & 0.046 & 0.041 & 0.037 & 0.033 & 0.030 & 0.027 & 0.025 \\
\hline$a_{3}$ & & 0.300 & 0.300 & 0.257 & 0.214 & 0.179 & 0.150 & 0.127 & 0.109 & 0.094 & 0.082 & 0.072 & 0.064 & 0.057 & 0.052 & 0.046 & 0.042 & 0.038 & 0.035 \\
\hline$a_{4}$ & & & 0.200 & 0.229 & 0.214 & 0.190 & 0.167 & 0.145 & 0.127 & 0.112 & 0.099 & 0.088 & 0.079 & 0.071 & 0.064 & 0.058 & 0.053 & 0.048 & 0.044 \\
\hline$a_{5}$ & & & & 0.143 & 0.179 & 0.179 & 0.167 & 0.151 & 0.136 & 0.122 & 0.110 & 0.099 & 0.089 & 0.081 & 0.074 & 0.067 & 0.061 & 0.056 & 0.052 \\
\hline$a_{6}$ & & & & & 0.107 & 0.143 & 0.150 & 0.146 & 0.136 & 0.126 & 0.115 & 0.106 & 0.096 & 0.088 & 0.080 & 0.074 & 0.068 & 0.063 & 0.058 \\
\hline $\mathrm{a}_{7}$ & & & & & & 0.083 & 0.117 & 0.127 & 0.127 & 0.122 & 0.115 & 0.108 & 0.101 & 0.093 & 0.086 & 0.080 & 0.074 & 0.068 & 0.064 \\
\hline$a_{8}$ & & & & & & & 0.067 & 0.097 & 0.109 & 0.112 & 0.110 & 0.106 & 0.101 & 0.094 & 0.088 & 0.083 & 0.077 & 0.073 & 0.068 \\
\hline$a_{9}$ & & & & & & & & 0.054 & 0.082 & 0.094 & 0.099 & 0.099 & 0.096 & 0.093 & 0.088 & 0.084 & 0.079 & 0.075 & 0.070 \\
\hline$a_{10}$ & & & & & & & & & 0.046 & 0.070 & 0.082 & 0.088 & 0.089 & 0.088 & 0.086 & 0.083 & 0.079 & 0.076 & 0.071 \\
\hline$a_{11}$ & & & & & & & & & & 0.039 & 0.060 & 0.072 & 0.079 & 0.081 & 0.080 & 0.080 & 0.077 & 0.075 & 0.071 \\
\hline$a_{12}$ & & & & & & & & & & & 0.034 & 0.052 & 0.064 & 0.071 & 0.074 & 0.074 & 0.074 & 0.073 & 0.070 \\
\hline$a_{13}$ & & & & & & & & & & & & 0.029 & 0.046 & 0.057 & 0.064 & 0.067 & 0.068 & 0.068 & 0.068 \\
\hline$a_{14}$ & & & & & & & & & & & & & 0.025 & 0.041 & 0.052 & 0.058 & 0.061 & 0.063 & 0.064 \\
\hline$a_{15}$ & & & & & & & & & & & & & & 0.022 & 0.037 & 0.046 & 0.053 & 0.056 & 0.058 \\
\hline$a_{16}$ & & & & & & & & & & & & & & & 0.019 & 0.033 & 0.042 & 0.048 & 0.052 \\
\hline$a_{17}$ & & & & & & & & & & & & & & & & 0.017 & 0.030 & 0.038 & 0.044 \\
\hline$a_{18}$ & & & & & & & & & & & & & & & & & 0.016 & 0.027 & 0.035 \\
\hline$a_{19}$ & & & & & & & & & & & & & & & & & & 0.014 & 0.025 \\
\hline $\mathrm{a}_{20}$ & & & & & & & & & & & & & & & & & & & 0.013 \\
\hline $\mathrm{S}(\mathrm{a})$ & 0.250 & 0.100 & 0.050 & 0.029 & 0.018 & 0.012 & 0.008 & 0.006 & 0.005 & 0.004 & 0.003 & 0.002 & 0.002 & 0.001 & 0.001 & 0.001 & 0.001 & 0.001 & 0.001 \\
\hline
\end{tabular}




\section{Simple Average of the CBE Derived Variables}

Iwueze et al. (2010) discussed the properties of the estimator based on the simple average (SAE: Simple Average Estimator) of the derived CBE variables given by

$$
\hat{b}^{(c)}=\frac{1}{(m-1)} \sum_{i=2}^{m} \hat{b}_{i}^{(c)}
$$

The mean and variance of (5.1) are:

$$
\begin{aligned}
E\left(\hat{b}^{(c)}\right)=b & \\
\operatorname{var}\left(\hat{b}^{(c)}\right)=\sigma_{\hat{b}^{(c)}}^{2} & =\frac{1}{(m-1)^{2}}\left\{\sum_{i=1}^{m-1} \operatorname{var}\left(\hat{b}_{i}^{(c)}\right)+2 \sum_{i<}^{m-1} \sum_{j}^{m-1} \operatorname{cov}\left(\hat{b}_{i}^{(c)}, \hat{b}_{j}^{(c)}\right)\right\} \\
& =\frac{1}{(m-1)^{2}}\left\{\sum_{i=1}^{m-1} \operatorname{var}\left(\hat{b}_{i}^{(c)}\right)-2 \sum_{i=1}^{m-2} \operatorname{cov}\left(\hat{b}_{i}^{(c)}, \hat{b}_{i+1}^{(c)}\right)\right\} \\
& =\frac{1}{(m-1)^{2}}\left\{(m-1)\left(\frac{2 \sigma^{2}}{s^{3}}\right)-(m-2)\left(\frac{2 \sigma^{2}}{s^{3}}\right)\right\} \\
& =\frac{2 \sigma^{2}}{(m-1)^{2} s^{3}}\{m-1-m+2\}=\frac{1}{(m-1)^{2}}\left(\frac{2 \sigma^{2}}{s^{3}}\right)
\end{aligned}
$$

The SA estimate (5.1) is also a linear unbiased estimator of the slope (b) parameter.

Comparing (4.6) and (5.3), we note that the difference between the variances of the SAE and the BLUE lies in the difference between $S(a)=\sum_{i=1}^{m-1} a_{i}^{2}-\sum_{i=1}^{m-2} a_{i} a_{i+1}$ for the BLUE and $\frac{1}{(m-1)^{2}}$ for the SAE. Figure 2 illustrates the differences.

The variance of the intercept (a) is given in Iwueze et al. (2010) as

$$
\operatorname{var}(\hat{\mathrm{a}})=\frac{\sigma^{2}}{\mathrm{n}}+\left(\frac{\mathrm{n}+1}{2}\right)^{2} \operatorname{var}(\hat{\mathrm{b}})
$$


where $\hat{b}=\hat{b}^{(c)}=\frac{1}{(m-1)} \sum_{i=2}^{m} \hat{b}_{i}^{(c)}$ for the SAE and $\hat{b}=T=\sum_{t=1}^{m-1} a_{i} \hat{b}_{i}^{(c)}$ for the BLUE. At $m=3, S(a)=\left(\frac{1}{m-1}\right)^{2}$ so that variances of the estimates of the slope are the same for BLUE and SAE

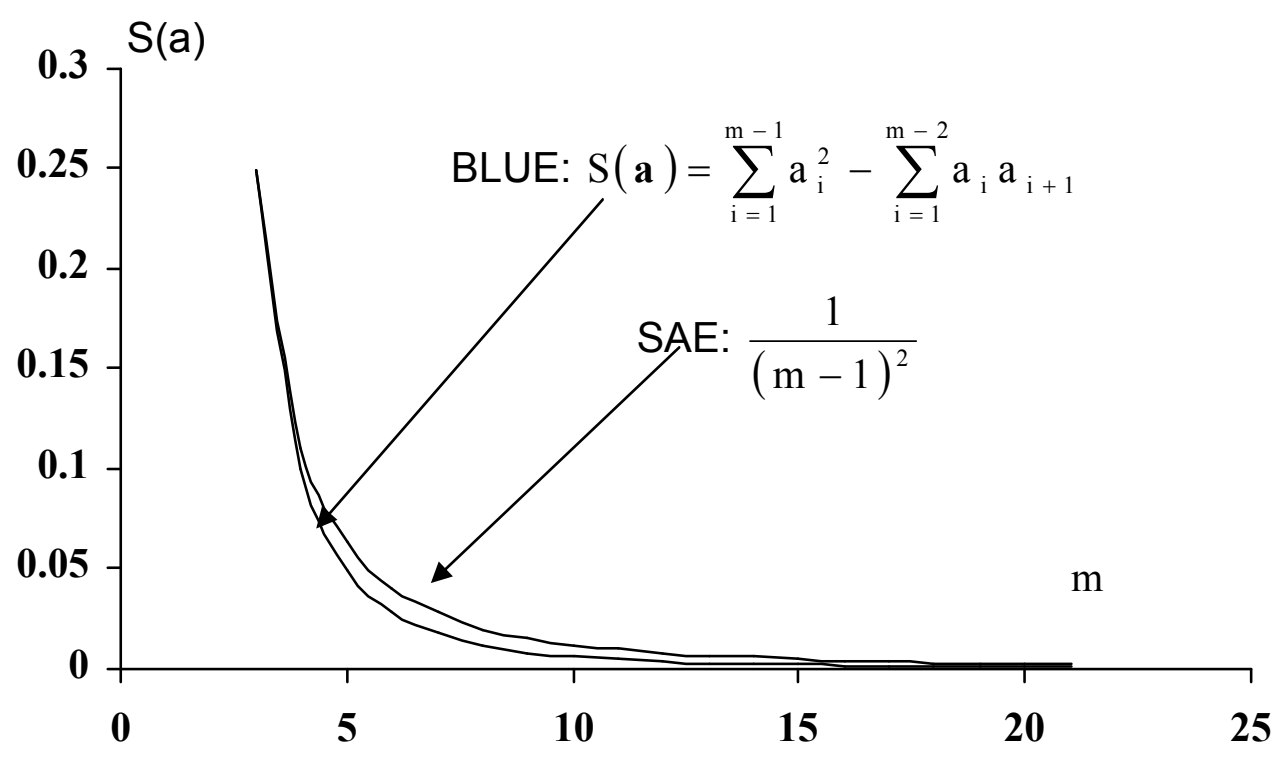

Figure 2: Plot of $\mathrm{S}(\mathbf{a})$ and $\frac{1}{(\mathrm{~m}-1)^{2}}$ against $\mathrm{m}$

\section{Empirical Examples}

The first example are simulations of $\mathrm{n}=4 \mathrm{~m}(\mathrm{~m}=8,11, \ldots, 18)$ observations from $\mathrm{X}_{\mathrm{t}}=\mathrm{a}+\mathrm{bt}+\mathrm{S}_{\mathrm{t}}+\mathrm{e}_{\mathrm{t}} \quad$ with $\quad \mathrm{a}=1.0, \mathrm{~b}=0.2, \mathrm{~S}_{1}=-1.5, \quad \mathrm{~S}_{2}=2.5, \mathrm{~S}_{3}=3.5$, $\mathrm{S}_{4}=-4.5$ and $\mathrm{e}_{\mathrm{t}} \sim \mathrm{N}(0,1)$. The properties of the BLUE were also determined and compared with those from the Least Squares Estimation (LSE) method and Simple Average Estimation (SAE) method of the Buys-Ballot derived variables.

As Table 4 shows, BLUE recovers the values of the slope and intercept used in the simulation better than the other two methods. The variances of the estimates of the slope and intercept are also smaller for the BLUE than for the other two methods.

The autocorrelation function (ac.f) of the residuals obtained after decomposition using the LSE, SAE and BLUE methods were used to confirm the adequacy of the fitted models. Diagnostic checks on the residuals are discussed in Box et al. (1994). 
The second example is the 32 consecutive quarters of U.S beer production, in millions of barrels, from first quarter of 1975 to the fourth quarter of 1982, and is listed as Series W10 in Wei (1990). In order to assess the forecasting performance of our models, we use only the first 30 observations of the series for model construction.

The estimates of the parameters using Least Squares Estimation method (LSE) are again determined and compared with those from the BLUE and SAE computed from the CBE derived variables. The computational procedure for the slope (b) is laid out in Table 5 while Table 6 gives the summary of the estimates.

Table 4: Summary of estimates of LSE, BLUE and SAE

\begin{tabular}{|c|c|c|c|c|c|c|c|c|c|c|}
\hline $\mathrm{m}$ & lethod & $\hat{a}$ & $\hat{b}$ & $\hat{\sigma}_{a}$ & $\hat{\sigma}_{b}$ & $\mathrm{~S}_{1}$ & $\mathrm{~S}_{2}$ & $\mathrm{~S}_{3}$ & $\mathrm{~S}_{4}$ & $\hat{\sigma}$ \\
\hline \multirow[b]{3}{*}{8} & $1 C 5$ & 309 & 101 & 1.261 & 0.067 & -1.322 & 2.534 & 2130 & -4.651 & non \\
\hline & & & & & & & & & & \\
\hline & _UE & 084 & 195 & 361 & 019 & -1.302 & 2.541 & 32 & 4.671 & 0.977 \\
\hline \multirow[b]{3}{*}{9} & & 174 & .191 & .196 & .056 & -1.538 & 3.086 & 3.108 & -4.656 & 0.875 \\
\hline & $A E$ & 185 & .190 & .413 & 0.021 & -1.539 & 3.086 & 3.108 & -4.655 & 0.876 \\
\hline & LUE & .965 & 0.202 & .313 & 0.015 & -1.521 & 3.092 & 3.102 & -4.673 & .861 \\
\hline \multirow[b]{3}{*}{10} & & & & & .047 & -1.347 & .403 & & -4.573 & .999 \\
\hline & & & 219 & & 020 & & .416 & & 14 & 1.019 \\
\hline & LUE & & 200 & 170 & 014 & & 347 & 3.574 & -4.406 & 1.004 \\
\hline \multirow[b]{3}{*}{11} & & .666 & .193 & 1.109 & 0.043 & -1.258 & 2.593 & 3.638 & -4.973 & 0.963 \\
\hline & & .762 & 0.211 & .411 & 0.017 & -1.231 & 2.602 & 3.629 & -5.000 & 0.966 \\
\hline & LUE & 987 & 0.201 & 306 & .012 & -1.246 & 2.597 & 3.634 & -4.985 & 0.957 \\
\hline \multirow[b]{3}{*}{12} & & & & & & -1.288 & 30 & & & \\
\hline & & & & & & -1.28 & 2.180 & 3.476 & -4.368 & 0.960 \\
\hline & LUE & 360 & .185 & .296 & .011 & -1.281 & 2.183 & 3.473 & -4.375 & 0.958 \\
\hline \multirow[b]{3}{*}{13} & & .199 & 193 & .026 & .034 & -1.273 & 2.683 & 3.644 & -5.054 & 0.947 \\
\hline & & & & & & & & & & 0.961 \\
\hline & $\mathrm{E}$ & & & & & & & & & \\
\hline \multirow[b]{3}{*}{14} & & & & & & 11 & 2.622 & 63 & -4.592 & .992 \\
\hline & & 726 & 210 & 407 & .013 & 380 & 2.627 & & -4.605 & 0.992 \\
\hline & BLUE & & 0.205 & .259 & .008 & -1.387 & 2.625 & 3.361 & -4.598 & 0.990 \\
\hline \multirow[b]{3}{*}{15} & & .265 & 0.191 & 0.847 & 0.024 & -1.465 & 2.393 & & -4.236 & 0.982 \\
\hline & & & & & & & & & & 1.006 \\
\hline & LUE & & & & & & & & -4.241 & 0.962 \\
\hline \multirow[b]{3}{*}{16} & & & & & & & 2.520 & 3.375 & -4.781 & 0.969 \\
\hline & SAE & & & & & & 2.518 & & -4.772 & 0.983 \\
\hline & BLUE & & & & & & 2.523 & & -4.787 & 0.967 \\
\hline \multirow[b]{3}{*}{17} & & & & & & & & & & \\
\hline & & & & & & & & & & 1.152 \\
\hline & BLUE & & & & & & & & -4.590 & 1.142 \\
\hline & LSE & 1.124 & 0.197 & 0.743 & 0.018 & -1.424 & 2.280 & 3.217 & -4.073 & 0.960 \\
\hline & & 0.919 & 1.044 & 0.382 & 0.010 & -1.415 & 2.283 & 3.213 & -4.081 & 0.962 \\
\hline & BLUE & 1.044 & 0.199 & 0.226 & 0.005 & -1.420 & 2.282 & 3.215 & -4.077 & 0.959 \\
\hline
\end{tabular}


Table 5: Buys-ballot table for U.S. beer production

\begin{tabular}{|c|c|c|c|c|c|c|c|c|c|}
\hline \multirow[b]{2}{*}{ Year } & \multicolumn{4}{|c|}{ Quarter } & \multirow[b]{2}{*}{$\bar{X}_{i .}$} & \multirow[b]{2}{*}{$\hat{\sigma}_{i .}$} & \multirow[b]{2}{*}{$\hat{b}_{i}^{(c)}$} & \multirow[b]{2}{*}{$a_{i}$} & \multirow[b]{2}{*}{$a_{i} \hat{b}_{i}^{(c)}$} \\
\hline & 1 & II & III & IV & & & & & \\
\hline 1975 & 36.14 & 44.60 & 44.15 & 35.72 & 40.15 & 4.88 & 0.2550 & 0.083 & 0.0212 \\
\hline 1976 & 36.19 & 44.63 & 46.95 & 36.90 & 41.17 & 5.43 & 0.3575 & 0.143 & 0.0511 \\
\hline 1977 & 39.66 & 49.72 & 44.49 & 36.54 & 42.60 & 5.76 & 0.5425 & 0.179 & 0.0971 \\
\hline 1978 & 41.44 & 49.07 & 48.98 & 39.59 & 44.77 & 4.97 & 0.3200 & 0.190 & 0.0608 \\
\hline 1979 & 44.29 & 50.09 & 48.42 & 41.39 & 46.05 & 3.95 & 0.6800 & 0.179 & 0.1217 \\
\hline 1980 & 46.11 & 53.44 & 53.00 & 42.52 & 48.77 & 5.35 & -0.0875 & 0.143 & -0.0125 \\
\hline 1981 & 44.61 & 55.18 & 52.24 & 41.66 & 48.42 & 6.34 & 0.6600 & 0.083 & 0.0548 \\
\hline 1982 & 47.84 & 54.27 & - & - & 51.06 & 4.55 & - & - & - \\
\hline$\overline{\mathrm{X}}_{. j}$ & 42.04 & 50.13 & 48.32 & 39.19 & 44.99 & & & & \\
\hline$\hat{\sigma}_{. j}$ & 4.42 & 4.07 & 3.46 & 2.78 & & 5.66 & & & \\
\hline
\end{tabular}

Table 6: Summary of estimates of LSE, BLUE and SAE for U. S beer production

\begin{tabular}{|l|l|l|l|l|l|l|l|l|l|}
\hline Method & $\hat{\mathrm{a}}$ & $\hat{\mathrm{b}}$ & $\hat{\sigma}_{\mathrm{a}}$ & $\hat{\sigma}_{\mathrm{b}}$ & $\mathrm{S}_{1}$ & $\mathrm{~S}_{2}$ & $\mathrm{~S}_{3}$ & $\mathrm{~S}_{4}$ & $\hat{\sigma}$ \\
\hline LSE & 39.099 & 0.380 & 1.790 & 0.101 & -2.692 & 5.018 & 3.592 & -5.918 & 1.244 \\
SAE & 38.955 & 0.390 & 0.564 & 0.033 & -2.297 & 5.403 & 3.207 & -6.313 & 1.307 \\
BLUE & 38.885 & 0.394 & 0.461 & 0.025 & -2.291 & 5.405 & 3.205 & -6.319 & 1.311 \\
\hline
\end{tabular}

Table 7: Comparison of the forecasts between models

\begin{tabular}{|c|c|c|c|c|c|c|c|c|c|}
\hline \multirow[b]{2}{*}{$\begin{array}{l}\text { Lead } \\
\text { time }\end{array}$} & \multirow[b]{2}{*}{$\begin{array}{l}\text { Actual } \\
\text { Value }\end{array}$} & \multicolumn{2}{|c|}{ Wei (1990) } & \multicolumn{2}{|c|}{ LSE } & \multicolumn{2}{|c|}{ SAE } & \multicolumn{2}{|c|}{ BLUE } \\
\hline & & $\begin{array}{c}\text { Forecast } \\
\text { Value }\end{array}$ & Error & $\begin{array}{c}\text { Forecast } \\
\text { Value }\end{array}$ & Error & $\begin{array}{c}\text { Forecast } \\
\text { Value }\end{array}$ & Error & $\begin{array}{c}\text { Forecast } \\
\text { Value }\end{array}$ & Error \\
\hline 1 & 52.31 & 54.38 & -2.07 & 54.48 & -2.17 & 54.24 & -1.93 & 54.31 & -2.00 \\
\hline 2 & 41.83 & 45.37 & -3.54 & 45.35 & -3.52 & 45.11 & -3.28 & 45.18 & -3.35 \\
\hline \multicolumn{2}{|l|}{$\begin{array}{l}\text { MPE } \\
\text { MSE } \\
\text { MAE } \\
\text { MAPF }\end{array}$} & \multicolumn{2}{|l|}{$\begin{array}{l}-6.2 \% \\
8.4 \\
2.8\end{array}$} & \multicolumn{2}{|l|}{$\begin{array}{l}-6.3 \% \\
8.6\end{array}$} & $\begin{array}{l}-5.8 \% \\
7.2 \\
2.6 \\
5.8 \%\end{array}$ & & $\begin{array}{l}-5.9 \% \\
7.6 \\
2.7 \\
5.9 \%\end{array}$ & \\
\hline
\end{tabular}

Wei (1990), ignoring the stochastic trend in the series, used 30 observations of the series for Integrated Autoregressive Moving Average (ARIMA) model construction. Based on the forecasting performance of his models, he settled on the model

$$
\left(1-\mathrm{B}^{4}\right) \mathrm{X}_{\mathrm{t}}=\underset{( \pm 0.99)}{1.49}+\left(1-\underset{( \pm 0.16)}{0.87} \mathrm{~B}^{4}\right) \mathrm{e}_{\mathrm{t}}
$$

with $\hat{\sigma}^{2}=2.39$.

The one step ahead and two step ahead forecasts, $\hat{\mathrm{X}}_{30}(\ell)$ for $\ell=1$ and 2 , from the forecast origin 30 are calculated for each estimation method. The forecast errors and the corresponding summary statistics used by Wei (1990) are shown in Table 7. With respect to Table 7, MPE is the Mean Percentage Error, MSE is 
the Mean Square Error, MAE is the Mean Absolute Error and MAPE is the Mean Absolute Percentage Error as defined in Wei (1990).

The results of Table 7 indicate that the SAE and BLUE give approximate results that are better than those given by the LSE and ARIMA in terms of forecasts. This example illustrates the fact that sometimes a simple descriptive model computed from the Buys-Ballot procedure may be preferred to the more complicated ARIMA and LSE methods in a series were all the methods are adequate in terms of the residuals.

\section{Concluding Remarks}

This study has examined the Best Linear Unbiased Estimator (BLUE) of the slope (b) of a linear trend-cycle component of time series computed from the Buys-Ballot derived variables defined by Iwueze and Nwogu (2004). The emphasis on the slope is based on the fact that estimates of the other parameters (intercept and seasonal indices) depend on it. The properties of the BLUE were also determined and compared with those from the Least Squares Estimation method (LSE) and Simple Average method (SAE) of the Buys-Ballot derived variables.

The results show that of the two derived variables (CBE and $F B E$ ), only the $C B E$ derived variable were found to be stationary (with constant mean and variance) but are correlated with only one significant autocorrelation coefficient at lag one. The derived variable from the FBE are non-stationary with constant autocorrelation coefficient at all lags. Hence, they are considered incapable, in their present state, to give any reliable estimate.

The variance of the BLUE for the slope (b) based on the CBE-derived variables was shown to depend on the sum of squares and cross-products $S(a)$ of the weights $\left(a_{i}\right)$ of the derived variables. The values of $S(a)$, in turn, depend on the number of periods $(\mathrm{m})$.

The variances of the estimates of the slope (the BLUE and SAE) are constant multiples of the variance of $\hat{b}_{\mathrm{i}}\left(\frac{2 \sigma^{2}}{\mathrm{~s}^{3}}\right)$. The multipliers are $\left(\frac{1}{m-1}\right)^{2}$ for SAE and $\mathrm{S}(\mathrm{a})$ for the BLUE. At $m=3, S(a)=\left(\frac{1}{m-1}\right)^{2}$ so that variances of the estimates of the slope are the same for BLUE and SAE. For $m>3$, the variances appear to decay exponentially as $\mathrm{m}$ increased.

The estimate of the slope based on simple average is only a particular case of the BLUE in which all the weights are equal $\left(i . e, a_{i}=\frac{1}{m-1}\right)$. The multipliers of 
$\frac{2 \sigma^{2}}{s^{3}}$ are, for every $m>3$, greater for SAE than BLUE. This ensures that the BLUE has minimum variance. As a consequence, the variances of the estimates of the intercept (a) are for every $m>3$, smaller for the BLUE than for the SAE. These are clearly supported by the results of the empirical examples shown in Table 4. Another important result is that (i) for $m>3$, the error variance is smaller for the BLUE than for the SAE and LSE and (ii) for most $m$ the estimates of the slope (b) and intercept (a) from BLUE are closer to the actual values used in the simulation than those from SAE and LSE.

Therefore, when using Buys-Ballot procedure for time series decomposition, it is recommended that when trend-cycle component is linear, the BLUE for the slope computed from the CBE-derived variable be used. This leads to more precise estimates of time series components.

\section{References}

1. Box, G. E. P. Jenkins, G. M. and Reinsel, G. C. (1994). Time Analysis, Forecasting and Control, $3^{\text {rd }}$ ed, Prentice-Hall, Englewood Cliffs N. J.

2. Chatfield, C. (2004). The Analysis of Time Series: An Introduction. $6^{\text {th }}$ ed., Chapman and Hall, London.

3. Draper, N. R. and H. Smith (1981). Applied Regression Analysis, John Wiley and Sons Inc., New York.

4. Iwueze, I. S. and E. C. Nwogu (2004). Buys-ballot estimates for time series decomposition, Global Journal of mathematical Sciences, Vol. 3, No. 2, pp. 83-98.

5. Iwueze, I. S. (2007). Some implications of truncating the $\mathrm{N}\left(1, \sigma^{2}\right)$ distribution to the left at zero, Journal of Applied Sciences, 7(2), pp. 189-195.

6. Iwueze, I. S. and A. C. Akpanta (2007). Effect of the logarithmic transformation on the trend-cycle component. Journal of Applied Sciences, 7(17), pp. 2414-2422.

7. Iwueze, I. S., A. C. Akpanta and H. C. Iwu (2008). Seasonal analysis of transformations of the multiplicative time series model, Asian Journal of Mathematics and Statistics, 1(2), pp. 80-89.

8. Iwueze, I. S., E. C. Nwogu and J. C. Ajaraogu (2010). Properties of the Buys-Ballot estimates when trend-cycle component of a time series is linear: additive case. International Journal of Mathematics and Computation, Vol. 8, No. S10, pp. 59-77.

9. Kendall, M. G. and Ord, J. K. (1990). Time Series, $3^{\text {rd }}$ ed., Charles Griffin, London.

10. Rohatgi, V. K. (1996). An Introduction to Probability Theory and Mathematical statistics, John Wily and sons, New York.

11. Wei, W. W. (1990). Time Series Analysis: Univariate and Multivariate Methods, Addison-Wesley Publishing Company, Inc. Redwood City. 